Sādhanā Vol. 36, Part 1, February 2011, pp. 35-51. (C Indian Academy of Sciences

\title{
Numerical modelling of failure of cement concrete using a unit cell approach
}

\author{
M D GHOUSE $^{1}, \mathrm{C} \mathrm{LAKSHMANA} \mathrm{RAO}^{1}$ and B N RAO ${ }^{2, *}$ \\ ${ }^{1}$ Department of Applied Mechanics, Indian Institute of Technology Madras, Chennai \\ 600 036, India \\ ${ }^{2}$ Structural Engineering Division, Department of Civil Engineering, Indian Institute \\ of Technology Madras, Chennai 600 036, India \\ e-mail: bnrao@iitm.ac.in
}

MS received 8 March 2010; revised 5 October 2010; accepted 26 October 2010

\begin{abstract}
In this paper, a unit cell based approach is followed, where a unit cell consisting of one aggregate surrounded by mortar matrix is used for numerical simulation of mechanical response of cement concrete. Unit cell approach is a simple mathematical approximation that helps us to simplify the simulation of mechanical response of multi-phase composites. To model the failure of matrix, brittle cracking model is used, where the entire fracture zone is represented by a band of micro cracked material. Current study involves; (a) failure analysis of the concrete unit cell when it is subjected to tensile loads, and (b) parametric study of variation of peak strength with shape and volume fraction of aggregate. In this study, circular and square aggregates at various orientations are modelled. The simulation results predict that the peak tensile stresses are not very sensitive to the volume fraction of aggregates, when the unit cell is subjected to tensile loads. This paper effectively demonstrates the power of unit cell model in simulating the nonlinear mechanical response of cement concrete when it is subjected to tensile loading.
\end{abstract}

Keywords. Particulate composites; mesolevel; unit cell approach; smeared cracking; quasi brittle materials; fracture of cement concrete.

\section{Introduction}

Many engineering materials are heterogeneous when observed at a microscale, even though at a large scale they are assumed to be homogeneous for analysis. Typical examples of materials which show heterogeneity at microscale are polymer-based composites, metal alloys, metal matrix composites, ceramics, concrete, polycrystalline materials, etc. Many particulate composites such as concrete, ceramics or metal-matrix composites have dispersion of particulates in

*For correspondence 
a matrix. In reinforced composites, stiff and strong second phase inclusions such as rock aggregate, glass, graphite, boron or aluminum oxide, etc. are added to matrices such as epoxy, steel, titanium or aluminum and cement mortar to enhance the strength, stiffness and wear resistance of the matrices. The mechanical behaviour of composite generally depends on the size, shape and properties of the second phase inclusions. Determination of the mechanical response of heterogeneous material is an essential problem in many engineering applications. Conducting experiments on a number of material samples, for various material properties, volume fraction of second phase particles and loading conditions in order to predict the response of a composite, is a difficult and expensive task. Therefore, analytical and numerical approaches are required to model the material at a lower length scale. The composite materials at lower length scale are heterogeneous. To predict the appropriate material response, these materials are modelled as multi phased materials explicitly. The length scale where a clear distinction of various phases is possible and is referred to as 'mesoscale'. To model material at mesoscale, a representative volume element (RVE) of the material is analysed. The RVE is defined as the minimum volume of laboratory scale specimen, such that the results obtained from this specimen can still be regarded as representative for a continuum (Van Mier 1996). Mesoscale model is analysed by taking RVE and applying necessary boundary conditions. This approach is known as 'unit cell approach'. Unit cell approach is employed in predicting the effective properties and nonlinear behaviour of composite materials (Kouznetsova et al 2001; Gitman et al 2008).

One of the materials that can be studied using the unit cell approach is the 'cement concrete'. Cement concrete can be visualized as a particulate composite at mesoscale consisting of mortar matrix and coarse aggregates. Mesoscale models of concrete are required to understand the failure process of concrete. Variation of volume fraction and properties of different phases, effect of the strength of concrete and the relationship between strength and microstructure of concrete can be studied using mesoscale models.

Mesolevel numerical simulation of the mechanical response of concrete has been performed using two general classes of elements. These are; (a) one-dimensional elements and (b) continuum elements. Each of these approaches is briefly explained below.

\subsection{Lattice models}

Damage processes of tensile loaded concrete specimen are studied by Schorn \& Rhode (1991) using framework model where small struts were used. Lattice models with beam elements are developed to numerically simulate the concrete material failure in tension (Schlangen \& Van Mier 1992). Fracture processes of concrete are studied using truss elements (Leite et al 2004; Li-Ping et al 2009; Andrea Spagnoli 2009). Each of these tensile truss elements are assumed to have failed when the tensile load in them reaches a limiting tensile load. A series of failed lattices would idealize the formation of a crack. Crack patterns predicted from lattice models matched well with the experimental observations. It was observed from literature (Vonk 1992) that the lattice model could capture well post peak softening behaviour and localization of deformation. Computational time consumed by lattice models is high and hence are not ideally suited to model the complete nonlinear response of concrete.

\subsection{Continuum models}

Continuum models are basically $2-\mathrm{d}$ or $3-\mathrm{d}$ models that idealize the material deformation as well as its failure in a continuum. These models work on 2-d and 3-d equilibrium equations and compatability conditions of a continuum. They also model failure using failure criteria 
defined for a continuum. They use 2-d or 3-d finite elements to model the deformation and failure processes. In such models, RVE of concrete is modelled using two-dimensional plane stress elements (Sadouki \& Wittmann 1988) or three-dimensional solid elements (Wriggers $\&$ Moftah 2006). The numerically generated concrete models were adopted to predict fracture in direct tension specimens. In these investigations, the fictitious crack approach is used to model (Hillerborg et al 1976) the failure in matrix phase and linear elastic model for the aggregates. A nonlinear finite element method suitable for mesoscopic study of concrete is developed to investigate the concrete failure in tensile loading (Kwan et al 1999). An algorithm is developed to model realistic RVE of concrete (Hafner et al 2006). Above discussed continuum model is developed for concrete in tensile and compressive loading. In this paper, a continuum model is used for numerically simulating mechanical behaviour of concrete under tensile loading using a unit cell approach.

\section{Unit cell for concrete}

A unit cell is a representative volume of material which repeats periodically to form macroscopic material. A unit cell should contain enough information of the microstructure and should be sufficiently small compared to the macroscopic structural dimensions. One of the objectives of unit cell approach is the prediction of macroscopic modulus of heterogeneous materials given the moduli of the individual phases and their geometric arrangement. The unit cell concept has also been applied to predict the nonlinear behaviour of the material (Kouznetsova et al 2001, Gitman et al 2008). There are few steps involved in modelling a unit cell.

\subsection{Step 1: Identification of appropriate unit cell for any given material}

The length of the unit cell differs for different materials. RVE size should roughly be $0.1 \mathrm{~mm}$ for metallic materials, $1 \mathrm{~mm}$ for polymers, $10 \mathrm{~mm}$ for woods and $100 \mathrm{~mm}$ for concrete (Lemaitre 1987). In the current work, a RVE of concrete is idealized as a square of $100 \mathrm{~mm}$ side consisting of a single aggregate surrounded by mortar matrix. Aggregate and mortar matrix are separated by a thin interface. The idealized unit cell that is used in numerical simulation is shown in the figure 1 . The unit cell with a single aggregate indicates that aggregate distribution is considered

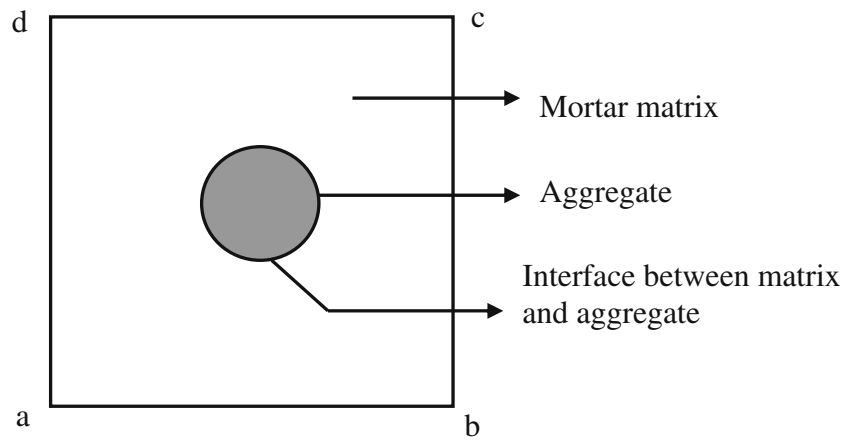

Figure 1. Idealized unit cell with constituent phases. 
as regular within the mortar matrix. This is an assumption made to simplify the computations associated with the problem, even if we use the assumed model to obtain bounds on concrete strength and its sensitivity to aggregate parameters.

\subsection{Step 2: Formulation of appropriate boundary conditions that would ensure periodicity of the unit cell}

Since current unit cell is surrounded by similar unit cells, deformation of current unit cell should be compatible with deformation of its surrounding unit cells. To ensure displacement compatibility of unit cell, periodic boundary conditions are applied. The periodic boundary conditions imply that the shape and orientation of two opposite edges remains identical during the deformation process. Periodic boundary conditions are discussed elaborately by Van der Sluis et al (2000). Periodic boundary conditions applied on unit cell are given by

$$
u_{i}^{l}-u_{i}^{r}=u_{i}^{a}-u_{i}^{b}, i=1,2, \ldots . n,
$$

where $u_{i}^{l}$ and $u_{i}^{r}$ are respectively the displacements of node $i$, located on the left and right edges of the unit cell as indicated in figure 1. Equation 1 indicates that the relative displacement of nodes on left and right edges must be equated to the relative displacement of some chosen master nodes $a$ and $b$, in order to ensure compatability between the unit cells. Periodic boundary conditions discussed in eq. 1 will ensure the periodicity in x-direction (horizontal direction).

\subsection{Step 3: Choice of appropriate constitutive model for different phases of unit cell}

The boundary value problem that is defined by the unit cell and its boundary conditions is solved using numerical tools like finite elements methods. At this stage constitutive models need to be chosen for (a) the deformation of materials that constitute each phase, (b) initiation and propagation of failure in any of the materials, and (c) initiation and propagation of failures at the interface. Detailed description of constitutive models adopted for different phases of the unit cell is discussed in the next section.

\section{Material models for constituents of a unit cell for cement concrete}

Cement concrete, which is considered as a particulate composite having coarse aggregates and cement mortar, can be modelled using unit cell approach. The details of material models used to create this model are described in this section.

\subsection{Modelling of aggregate}

Coarse aggregates represent around $40-50 \%$ of the concrete volume. Gravels constitute majority of coarse aggregate used in concrete followed by the crushed stones. Shape of the aggregate particles depends on aggregate type. In general, gravel aggregates have rounded and angular shapes while crushed stone aggregates have very fine particles, which will blend with the cement 
mortar. In the present work, circular and square shapes (with different orientations) of aggregate are chosen for parametric study. These aggregates are assumed to have a linear-isotropic material response, where no separation takes place.

\subsection{Modelling of mortar matrix}

Mortar matrix shows softening behaviour after reaching the tensile strength. This softening can be captured using stress-crack opening curves. This softening behaviour is due to formation of a fracture process zone within the mortar matrix. Material in this zone softens progressively due to the formation of microcracks. This softening behaviour is localized in a fictitious crack, where stress transfer normal to the crack can still take place. The stress transfer capacity as function of the crack width is given by the strain-softening relation. The area under stresssoftening diagram is equal to fracture energy $\left(G_{f}\right)$ of mortar matrix. Fracture energy of matrix is generally determined experimentally using the notched specimen loaded in flexure. The value for $G_{f}$ is obtained by computing the area under the load-deflection curve and dividing it by the net area of cross-section of the specimen above the notch (Mehta \& Monteiro 1993). In the current work, $G_{f}$ is calculated form CEB-FIP model code 1990 which recommends the use of the following expression for fracture energy (Mehta \& Monteiro 1993):

$$
G_{f}=\alpha_{f}\left(f_{c m} / f_{c m o}\right)^{0.7}
$$

where $\alpha_{f}$ is a coefficient, which depends on maximum aggregate size and $f_{\text {cmo }}$ is a constant. Values of $\alpha_{f}$ and $f_{c m o}$ are 0.02 and $10 \mathrm{MPa}$ respectively as recommended by CEB-FIP model code 1990 and $f_{c m}$ is compressive strength of matrix. Stress-crack opening relation is calculated from CEB-FIP model code 1990 which recommends a bilinear stress-crack opening relationship given by

$$
\begin{aligned}
& \sigma_{c t}=\left\{\begin{array}{l}
f_{t}\left(1-0.85 \frac{w}{w_{1}}\right) \quad \text { for } 0.15 f_{t} \leq \sigma_{c t} \leq f_{t}, \\
\frac{0.15 f_{t}}{w_{c}-w_{1}}\left(w_{c}-w\right) \text { for } 0 \leq \sigma_{c t} \leq 0.15 f,
\end{array}\right. \\
& \text { where } w_{1}=\frac{2 G_{f}}{f_{t}}-0.15 w_{c} \text { and } w_{c}=\beta_{f} \frac{G_{f}}{f_{t}},
\end{aligned}
$$

where $f_{t}$ is direct tensile strength of the matrix in $\mathrm{MPa}, \sigma_{c t}$ is cracked tensile stress in $\mathrm{MPa}$, $w_{1}$ is crack opening, $w_{c}$ is crack opening for $\sigma_{c t}=0$ and $\hat{a}_{f}$ is coefficient dependent on maximum aggregate size whose value is 8, as recommended by CEB-FIP model code 1990.

In this current work, a brittle crack model is adopted to model the behaviour of mortar matrix. This model is based on fictitious crack model proposed by Hillerborg et al (1976). This model uses a smeared crack approach to represent the discontinuous brittle behaviour in concrete. It does not track individual 'macro' cracks. In lieu of this, constitutive calculations are performed independently at each material point of the finite element model. The presence of cracks enters into these calculations as the way in which the cracks affect the stress and material stiffness associated with the material point. A simple Rankine criterion is used to detect crack initiation (Abaqus documentation 2007). This criterion states that a crack forms when the maximum 
principal tensile stress $\sigma_{1}$ exceeds the tensile strength $f_{t}$ of the brittle material and is given by the expression

$$
\left(\sigma_{1}\right)_{\text {matrix }} \geq\left(f_{t}\right)_{\text {matrix }} .
$$

As soon as the Rankine criterion for crack formation is satisfied, the algorithm assumes that a first crack has formed. The crack surface is taken to be normal to the direction of the maximum tensile principal stress. Post failure behaviour is specified as a stress-crack opening curve which is shown in figure 2. An important feature of the cracking model is that, whereas crack initiation is based on Mode I fracture only, postcracked behaviour includes Mode II as well as Mode I. The Mode II shear behaviour is based on the common observation that the shear behaviour depends on the amount of crack opening (Abaqus documentation 2007). More specifically, the cracked shear modulus is reduced as the crack opens, as given in the expression below:

$$
\begin{aligned}
& G_{c}=\rho\left(e_{n n}^{c k}\right) G, \\
& \text { where } G_{c}=\text { post crack shear modulus } \\
& G=\text { uncracked shear modulus } \\
& e_{n n}^{c k}=\text { crack pening strain } \\
& \rho=\text { shear retention factor. }
\end{aligned}
$$

\subsection{Modelling of interfacial transition zone}

Interface transition zone (ITZ) is the interface between mortar matrix and aggregate. In most problems dealing with interface of two materials, the ITZ is postulated to play an important role in the mechanics of failure of materials (Sadouki \& Wittmann 1988; Wang et al 1999). To investigate the effect of interface numerically, a $100 \mu \mathrm{m}$ interface is introduced between aggregate and matrix. Constitutive behaviour of interface is assumed to be similar to that of the

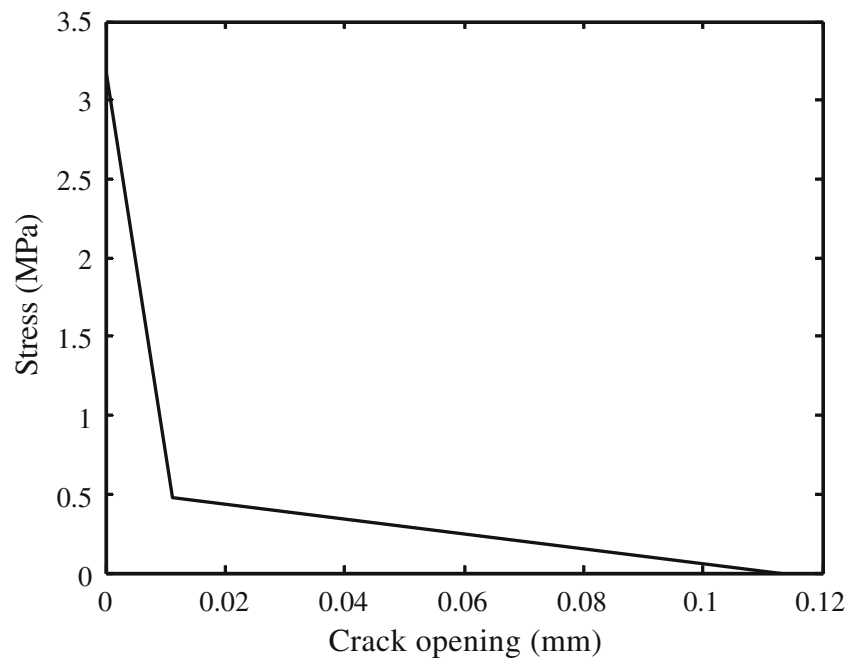

Figure 2. Stress-crack opening curve for mortar matrix. 
matrix material. The interface is assumed to have a linear elastic response till the peak stress which is predicted by Rankine's model. After reaching the peak stress, post-peak behaviour of interface is governed by a linear curve. Area under the post-peak curve is fracture energy of the interface. Material properties for interface are obtained from Van Mier (1996). Interface properties used for the simulation are given in table 1.

A commercially available software program (ABAQUS 6.8ef) is used for the finite element analysis. Four-noded plane strain elements (CPE4R) are used to mesh all three phases of unit cell. Discretized unit cell with plane strain elements is shown in figure 3.

\section{Validation of finite element mesh for elastic analysis with analytical solution}

Analytical solutions for elastic analysis of circular inclusion in a semi infinite plate under tensile load has been derived (Shioya 1967). This analysis is carried out by applying the bipolar coordinates with the help of a method of perturbation. In this analysis, inclusion is assumed to be perfectly bonded to the matrix material. The maximum principal stress on the common boundary using this method is given by the expression

$$
\sigma_{\max }=T\left\{\frac{1}{(1+v)+(1-\bar{v}) \Gamma}+\frac{2}{(3-v)+(1+\bar{v}) \Gamma}\right\}, \Gamma=\frac{E}{\bar{E}},
$$

where $T$ is the applied tensile stress at infinity, $v$ and $\bar{v}$ are Poisson's ratio of matrix and inclusion respectively, $E$ and $\bar{E}$ are Young's modulus of matrix and inclusion respectively. When plate is subjected to a tensile stress of $1 \mathrm{MPa}$, and substituting elastic properties given in table 1 and in equation 6, maximum principal stress on the common boundary is 1.291 MPa.

To check the validity of numerical model, the simulation results from the FEM model are compared with the results predicted by Shioya (1967). Numerical simulations are performed under plane stress condition. A $20 \mathrm{~mm}$ diameter inclusion is embedded in $100 \times 100 \mathrm{~mm}$ square plate. Elastic properties adopted for numerical simulation are given in table 1 . The maximum principal stress on the common boundary predicted from numerical simulation is 1.29 MPa. The difference in maximum principal stress predicted by numerical simulation is $0.1 \%$. Numerical prediction shows a close agreement with the analytically predicted results. This validates the current numerical model in the elastic region. It must be noted that further simulations that are presented in the subsequent sections are done for a nonlinear analysis, where failure is simulated. Principal stress contours for the linear analysis are plotted in figure 4.

Table 1. Material properties used for numerical simulation.

\begin{tabular}{lccc}
\hline & Mortar matrix & Aggregate & ITZ \\
\hline Young's modulus $(\mathrm{GPa})$ & 28.7 & 86.7 & 40 \\
Poisson's ratio & 0.27 & 0.3 & 0.3 \\
Fracture energy $\left(\mathrm{Nm} / \mathrm{m}^{2}\right)$ & 44.85 & - & 30 \\
Tensile strength $(\mathrm{MPa})$ & 3.17 & - & 2.0 \\
Density $\left(\mathrm{kg} / \mathrm{m}^{3}\right)$ & 2162 & 1650 & 2000 \\
\hline
\end{tabular}




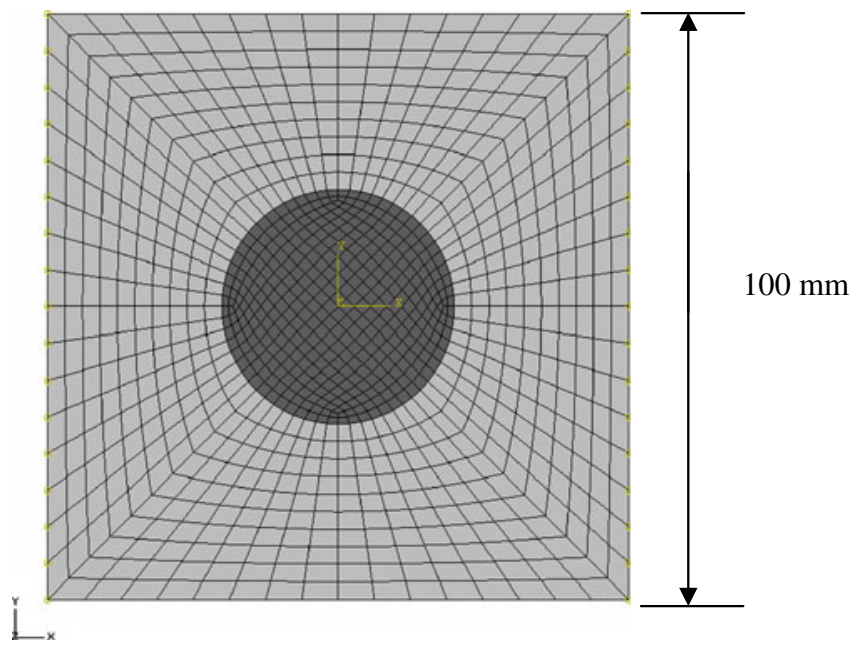

Figure 3. Discretized unit cell.

\section{Numerical simulation of tensile response of concrete using unit cell model}

In this work, numerical simulation consists of (i) failure analysis of concrete unit cell when it is subjected to tensile loads; and (ii) parametric study for variation of tensile strength with shape and volume fraction of aggregates. The results of the unit cell simulation are assumed to represent the mechanical behaviour of concrete (as obtained from standard test specimens), since the unit cell is assumed to represent RVE.
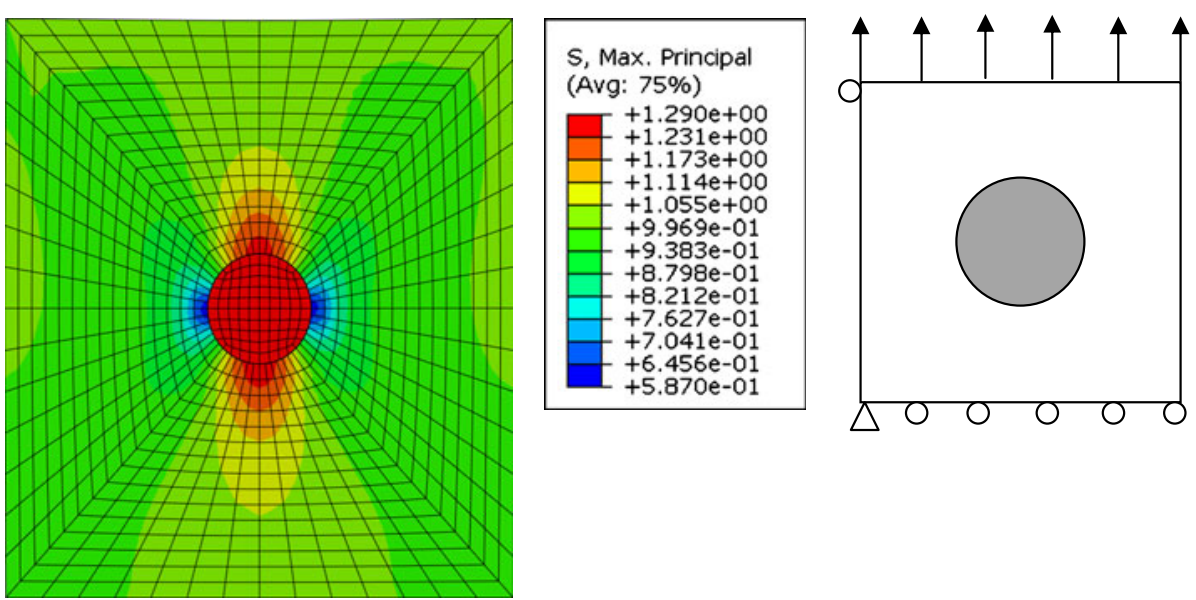

Figure 4. Principal stress contours in MPa of linear analysis conducted to compare numerical results with analytical solution. 


\subsection{Justification of tensile response predictions using a single aggregate unit cell}

In this work, a single aggregate (with circular and square shape) is taken in a unit cell. This model assumes that the distribution of aggregates is regular within the matrix. In order to validate the suitability of this assumption (in simulating the mechanical response of the material observed in experiments), a study is conducted to compare the mechanical response of unit cell with multiple aggregates distributed in the domain. In the numerical simulation unit cell with $12.5 \%$ aggregate volume fraction is used for both unit cells having a single aggregate and multiple aggregates. Material models described in section 3, are assigned to aggregate and matrix respectively. Material properties adopted for two phases are given in table 1 . In regular unit cell a single aggregate occupies the total $12.5 \%$ volume whereas in distributed cell volume fraction is divided among three circular aggregates of equal size. Positions of three aggregates are decided arbitrarily, unit cell with distributed aggregates is shown in figure $5 \mathrm{~b}$. Numerical simulations of both unit cells are conducted for tensile loading. Maximum principal stress contours obtained from numerical simulations are shown in figure 5. Figure 6 shows the stress-strain response of both the unit cells obtained for tensile loading. It can be observed that there is no difference in both stress-strain curves within linear region. Nonlinear behaviour starts at $80 \%$ of peak stress. At this stage there is a small shift in the response of distributed unit cell. The difference in peak stress is $0.3 \%$. Post peak curve of random unit cell is above the regular unit cell. This indicates that the response of distributed unit cell is more ductile than regular unit cell. Kouznetsova et al (2001), have compared the unit cell with regular and distributed voids. Results simulated by
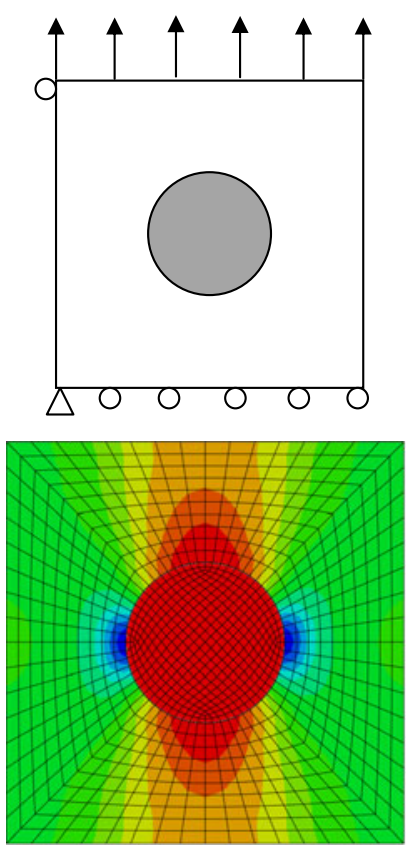

(a)
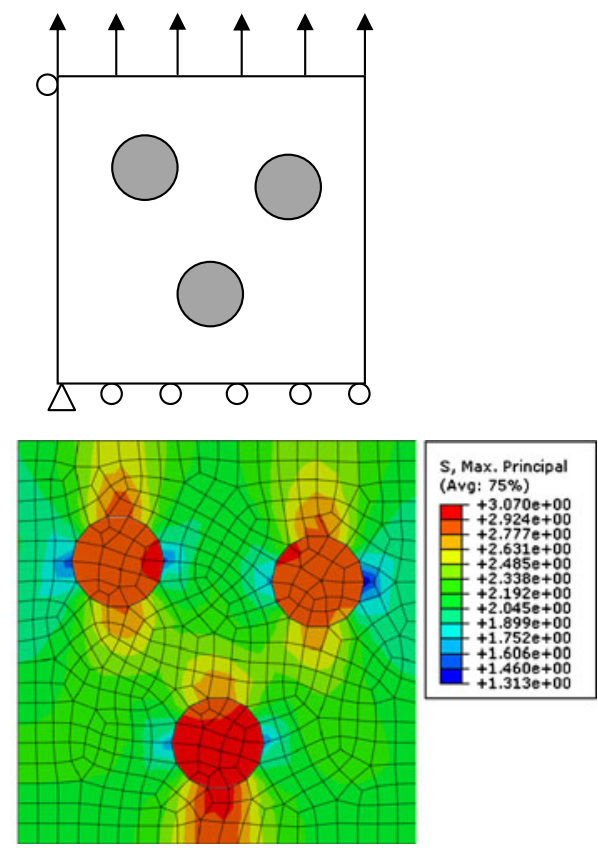

(b)

Figure 5. Maximum principal stress contours in MPa (a) regular unit cell with single aggregate, (b) Distributed unit cell with three aggregates. 


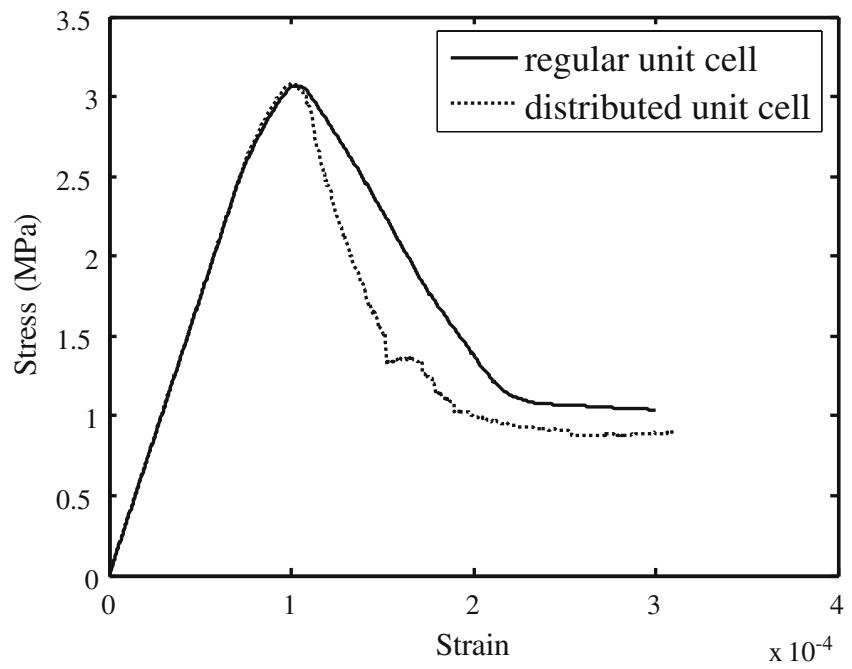

Figure 6. Stress-strain response of regular unit cell with single aggregate and distributed unit cell with three aggregates.

these authors indicated that the material response in regularly distributed unit cell is $2 \%$ higher in elastic zone and it reaches up to $10 \%$ in plastic zone. From the results of Kouznetsova et al (2001) and the simulation done by the author, it can be concluded that assumption of regular distribution of aggregate in cement concrete, is a reasonable assumption that will yield a good bound on the peak stresses and their sensitivity to geometric parameters of model. The assumption of regular distribution of aggregates not only simplifies the problem, but also assist in studying the effect of aggregate shape and size on overall failure behaviour of concrete. Hence, in this study a regular unit cell with single circular and square aggregate is adopted.

\subsection{Unit cell with Interfacial Transition Zone (ITZ)}

To simulate the effect of interface numerically, $100 \mu \mathrm{m}$ interface is introduced between aggregate and matrix (Mehta \& Monteiro 1993). Material model adopted for interface modelling is described in section 3. Properties of ITZ are taken from Van Mier (1996). Material properties adopted for the simulations are given in table 1. Simulations are conducted on unit cell with $12.5 \%$ volume fraction of aggregate, in tension.

Numerical simulations are conducted on unit cell for tensile loading. Stress-strain response obtained for three-phase and two-phase unit cell are compared in figure 7. Predictions from the simulations show there is decrease of $7 \%$ in peak stress when ITZ is added. Except for the postpeak behaviour, there is not much difference in the response of the material. Post-peak failure for unit cell without ITZ is more brittle when compared to unit cell with ITZ. This can be due to the fact that the load is initially taken by ITZ and then by mortar matrix, after the failure of ITZ. Simulations with ITZ are more complex and consumed 10 times more computational time compared to two-phase simulations. Since, the main aim of this work is to study the effect of aggregate volume fraction and shape on peak stress, moreover the difference in predicted peak stress by both unit cell models (with and without ITZ) is very small, all further parametric studies are restricted to unit cells without the ITZ. 


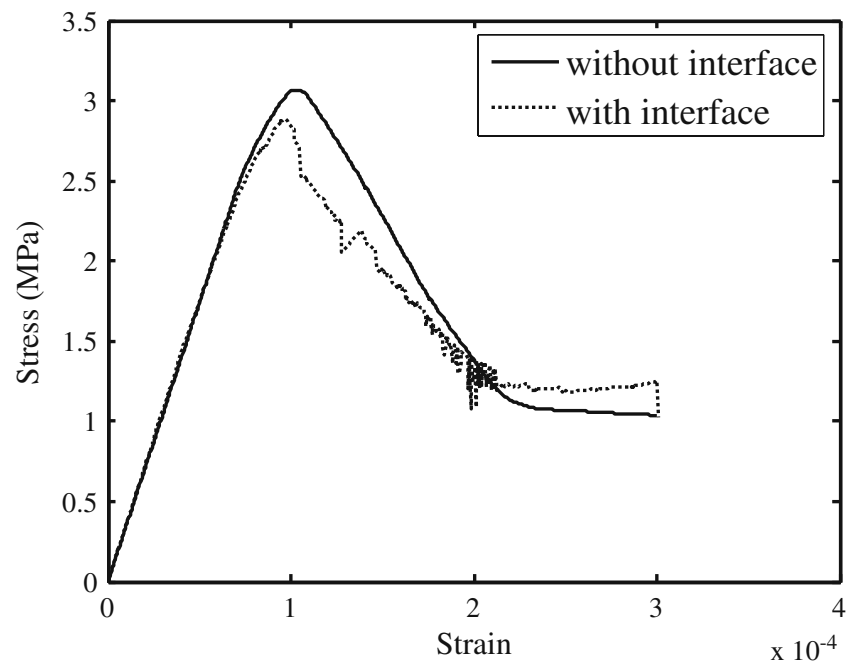

Figure 7. Comparison of stress-strain plot for two and three phase unit cell for tensile load.

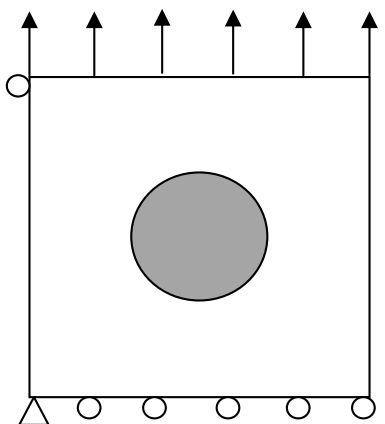

(a)

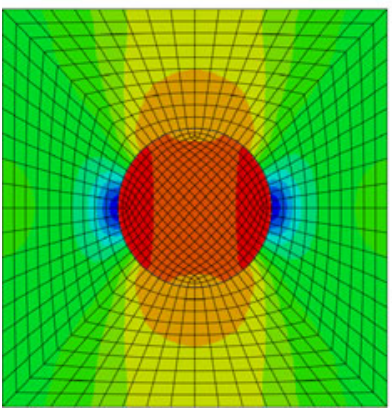

(c)

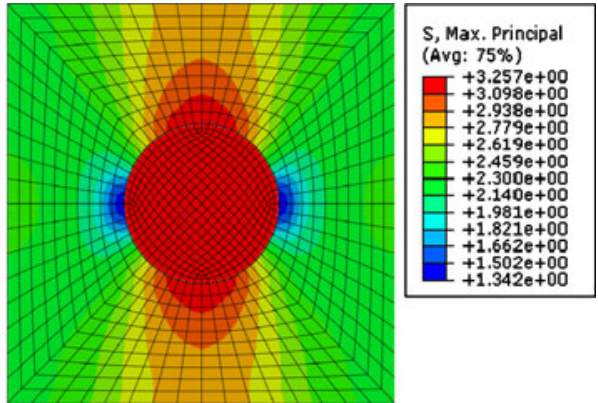

(b)
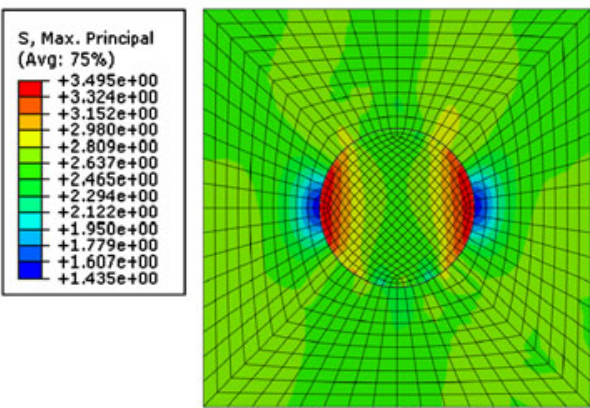

(d)

Figure 8. Maximum principal stress contours in MPa of unit cell with circular aggregates in tension at (a) unit cell under tension, (b) pre-peak load, (c) peak load and (d) post-peak load. 


\subsection{Mechanical response of two phase unit cell model of cement concrete subjected to tensile loading}

5.3a Tensile response with circular aggregates: The unit cell with circular aggregate is simulated for tensile loading. Principal stress contours predicted from the numerical simulation are shown in figure 8. A stress concentration zone is formed around aggregate, high stress region can be observed above and below the aggregate (red zone) in figure 8 . Failure is initiated in the elements falling under these high stress zones. When these elements reach tensile strength of the matrix, first crack initiates in the element and stress across the crack follow the stress-crack opening relation given in equation 3 . After this point of load, there will be an unloading observed in the global stress-strain behaviour as shown in figure 9.

It is observed from figure 9 that the stress-strain response is linear up to $90 \%$ of the load, nonlinear response starts when the first crack initiated. Even after the initiation of first crack, unit cell can take load up to the peak stress. After reaching the peak stress, unloading can be observed, at this point many elements are cracked and according to stress-crack opening relation, load carrying capacity of the cracked elements is reduced which result in overall softening response of the material. Above discussed results are for $12.5 \%$ volume fraction of aggregate, which corresponds to $40 \mathrm{~mm}$ diameter of aggregate.

Numerical simulations are conducted for varying aggregate volume fraction. Figure 10 shows the variation of peak stress with volume fraction. There is a decrease of $1.1 \%$ in peak stress when the volume fraction is increased from $2 \%$ to $5.3 \%$, an increase of $2.3 \%$ when volume fraction is increased from $5.3 \%$ to $12.5 \%$ and then a decrease of $4.7 \%$ in peak stress when volume fraction is increased from $12.5 \%$ to $28.3 \%$.

5.3b Tensile response with square aggregate: Most commonly used aggregates are angular which cause stress concentration at the sharp corners. Study is conducted on unit cell with square aggregate to understand the effect of angularity. Since, within the mortar matrix aggregates are

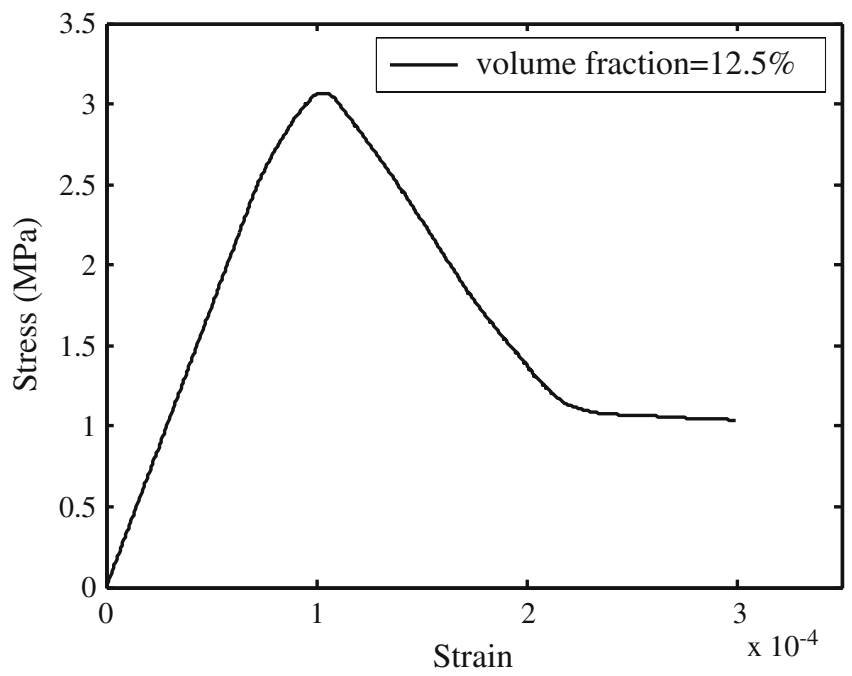

Figure 9. Stress-strain response of concrete unit cell (circular aggregate) for tensile loading. 


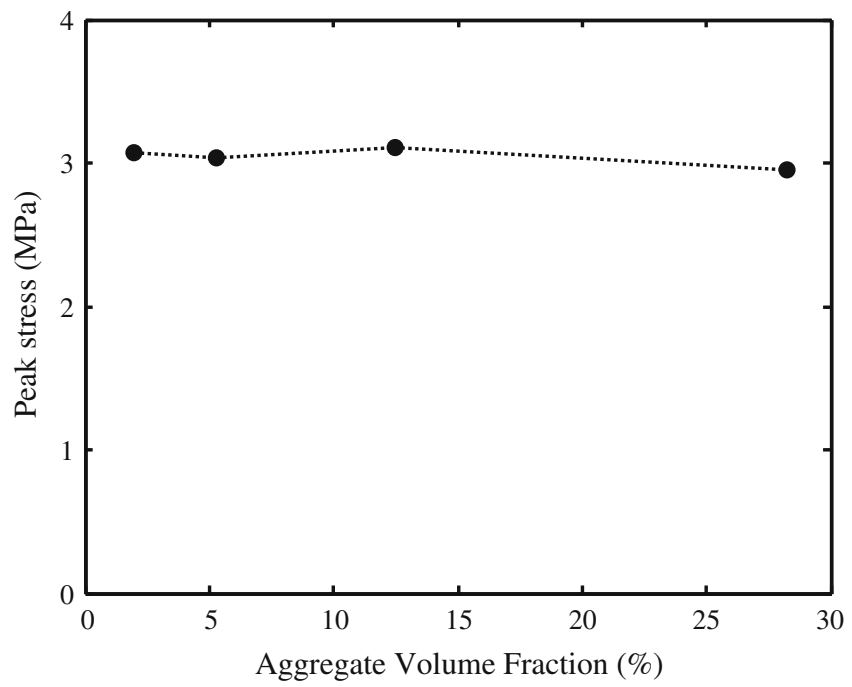

Figure 10. Variation of peak stress with aggregate volume fraction (circular aggregate).

dispersed and can be oriented in any direction, square aggregates are oriented with respect to its diagonal as shown in figure 11.

Figure 12 shows the maximum principal stress contours of the unit cell with square aggregate oriented at $30^{\circ}, 45^{\circ}$ and $90^{\circ}$. Principal stress contours shown in figure 12 are captured before failure, when principal stresses have reached tensile strength of the matrix. Red zones above and below the aggregate indicate the high stress zones. Failure patterns are same as observed for circular aggregate. Figure 13 shows the stress-strain response of unit cell with square aggregates.

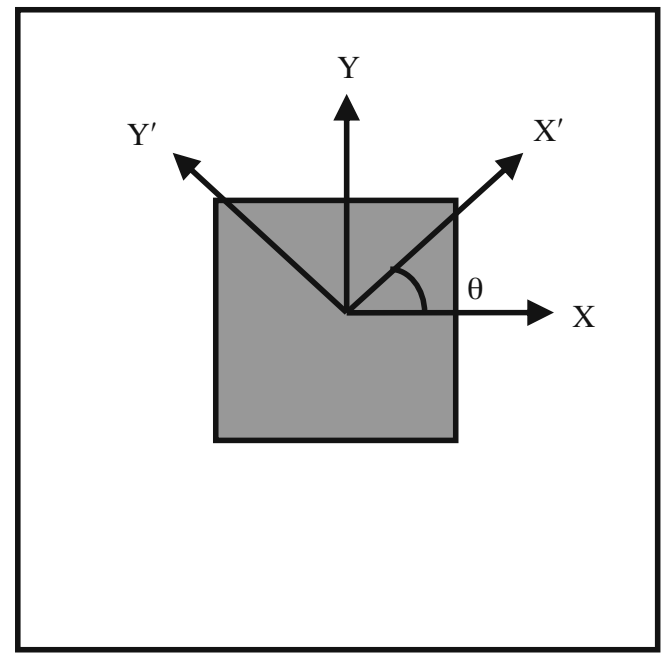

Figure 11. Unit cell with square aggregate oriented at an angle $\theta$ with respect to diagonal. 
(a)

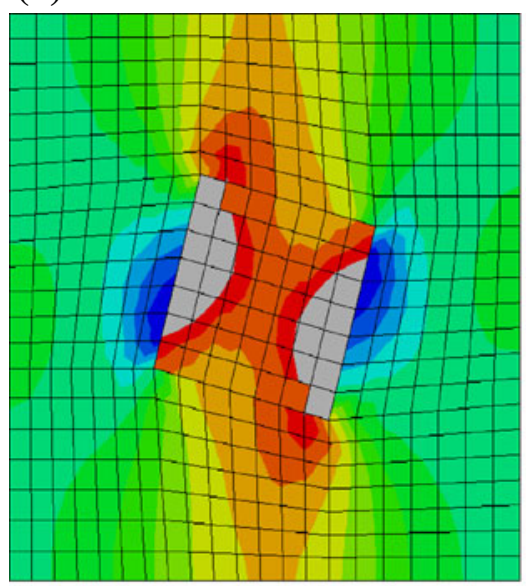

(b)

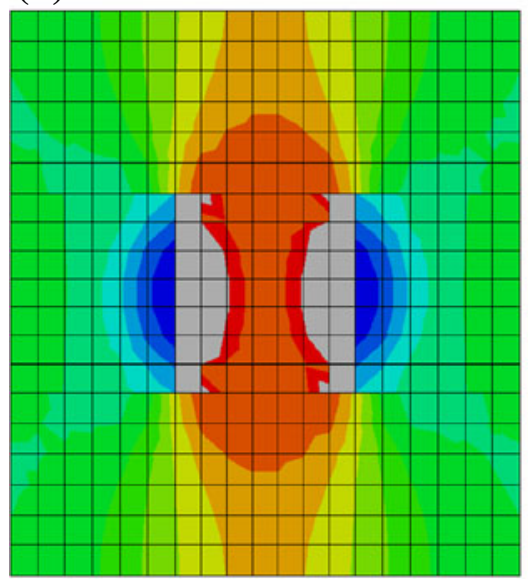

(c)

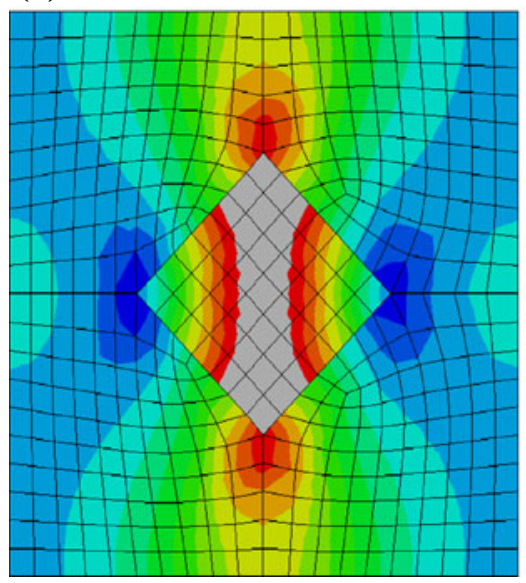

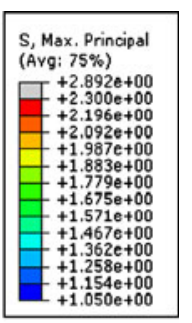

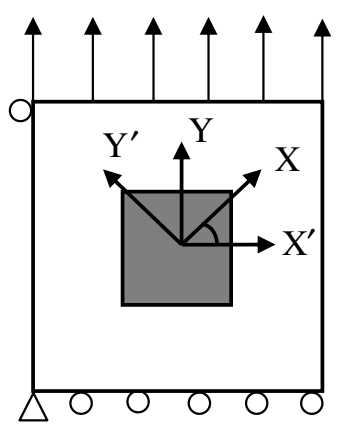

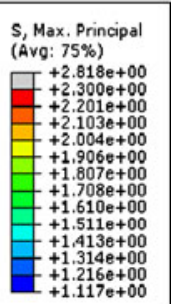

Max, Principal (Avg: $75 \%$ )
(A) (Avg: $75 \%$ ) $+2.300 \mathrm{e}+00$ $+2.219 \mathrm{e}+00$ $+\begin{array}{r}+2.138 \mathrm{e}+00 \\ +\end{array}$ $+2.057 \mathrm{e}+00$ $+1.895 \mathrm{e}+00$ $+1.814 \mathrm{e}+00$ $+1.733 \mathrm{e}+00$ $+1.652 \mathrm{e}+00$ $+1.571 \mathrm{e}+00$

$+1.490 \mathrm{e}+00$

$+1.409 \mathrm{e}+00$
$+1.328 \mathrm{e}+00$

Figure 12. Maximum principal stress contours in MPa for different orientation of the square aggregates (a) $30^{\circ}$, (b) $45^{\circ}$ and (c) $90^{\circ}$ in tension. 


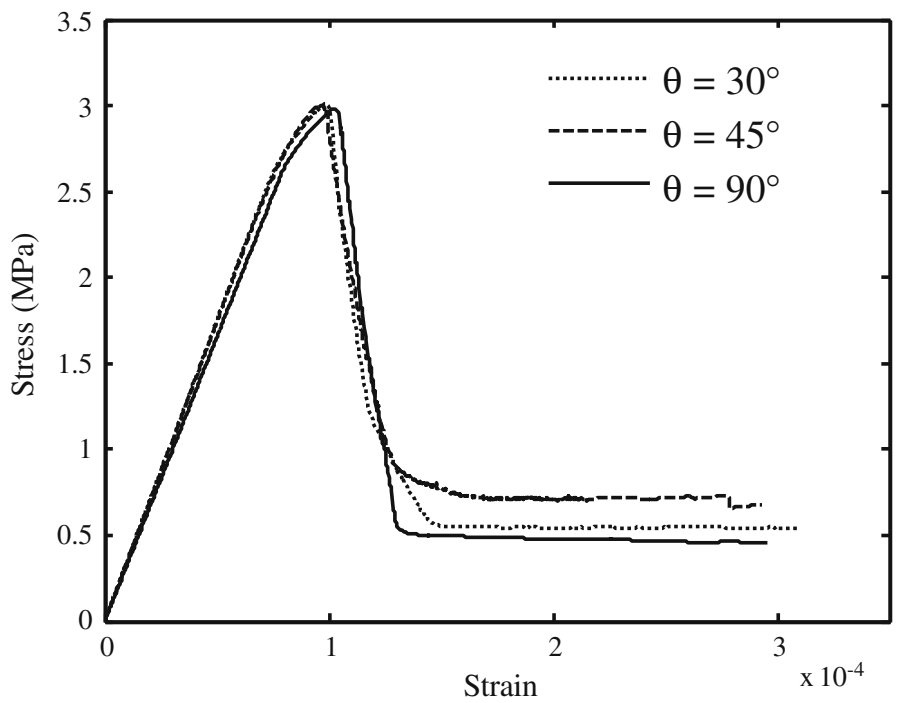

Figure 13. Stress-strain response of concrete unit cell (square aggregate) with $12.5 \%$ aggregate volume fraction for tensile loading.

For all square orientations, simulations are conducted for varying aggregate volume fraction and results are plotted in figure 14. There is a decreasing trend in peak stress with aggregate volume fraction, however, decrease is very small. The peak stresses predicted by unit cell with square aggregate is approximately similar for all orientations. Here we can conclude that orientation of aggregate have very small impact on the behaviour of concrete for tensile loading.

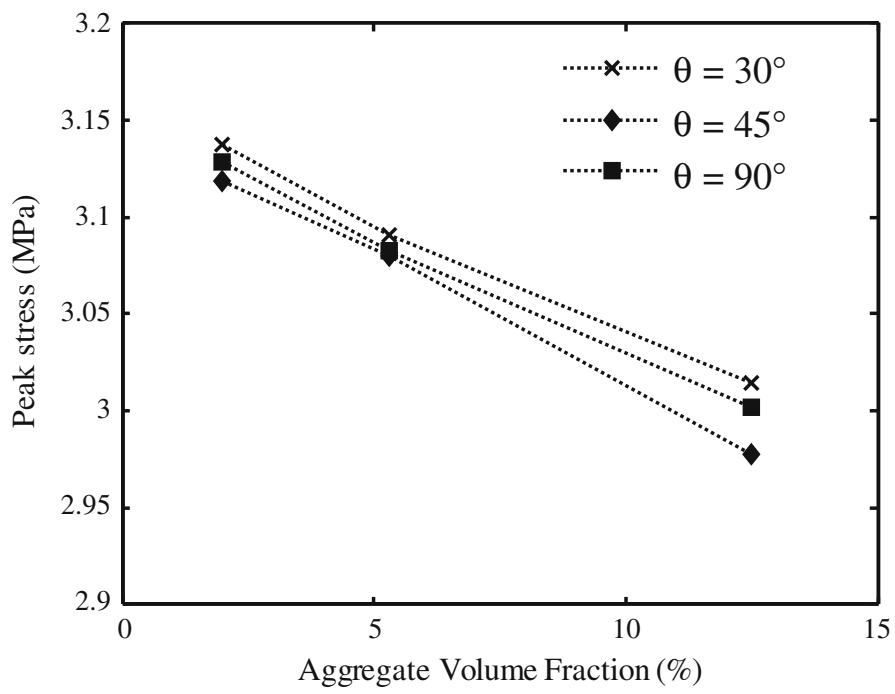

Figure 14. Variation of peak stress with aggregate volume fraction (square aggregate). 


\section{Conclusions}

In this work a meso-mechanical model based on unit cell approach is established to model the heterogeneous materials like concrete. A detailed description of unit cell approach and mechanical modelling of constituent phases is explained. Interface between the aggregate and matrix phases is modelled explicitly to study the effect of interface on the mechanical behaviour of the material. A parametric study is conducted for various sizes and shapes of aggregate on mechanical behaviour of concrete in tension. In this study, circular and square aggregates at various orientations are modelled. Numerical simulations are performed for uniaxial tensile loading on unit cell model of concrete. Main conclusions from this work are:

(i) Under tensile loads the difference in peak stresses predicted by unit cell without interface, is marginally higher than unit cell with interface but the computational time consumed for numerical simulations with interface is 10 times higher.

(ii) Variation of tensile strengths with volume fraction of aggregates, as predicted in the current simulation, is marginal.

(iii) The peak stress predicted for unit cell with circular aggregates is marginally higher than unit cell with square aggregate.

This work clearly demonstrates the power of the unit cell concept in the numerical simulation of the mechanical response of a brittle material like concrete. It is postulated that any structural variation in concrete such as addition of fibres, presence of huge voids, or the presence of nano scale second phase particles, can be effectively simulated by appropriately modifying the unit cell associated with any given material.

\section{Nomenclature}

$u_{i}^{l}$ Displacement of node $i$ on the left edge of unit cell

$u_{i}^{r}$ Displacement of node $i$ on the right edge of unit cell

$\alpha_{f}$ Coefficient which depends on maximum aggregate size

$f_{c m}$ Compressive strength of mortar matrix

$f_{t}$ Tensile strength of mortar matrix

$\sigma_{c t}$ Cracked tensile stress

$w_{c}$ Critical crack opening separation

$l_{c h}$ Characteristic length

$G_{f}$ Fracture energy

$\sigma_{1}$ Maximum principal stress

$G_{c}$ Cracked shear modulus

$G$ Shear modulus

$e_{n n}^{c k}$ Crack opening strain

$\rho$ Shear retention factor

$E$ Young's modulus of mortar matrix/plate

$\bar{E}$ Young's modulus of inclusion/aggregate

$v$ Poisson's ratio of mortar matrix/plate

$\bar{v}$ Poisson's ratio of inclusion/aggregate

$T$ Applied tensile stress at infinity

First author acknowledges the financial support of the Ministry of Human Resource Development (MHRD), India for providing half time teaching and research assistantship. 


\section{References}

ABAQUS 6.7ef 2007 Documentation, Dassault Systèmes December 12

Gitman I M, Askes H, Sluys L J 2008 Coupled-volume multi-scale modelling of quasi-brittle material. Eur. J. Mech. A/Solids, 27: 302-327

Guo L-P, Carpinteri A, Roncella R, Spagnoli A, Sun W, Vantadori S 2009 Fatigue damage of high performance concrete through a 2D mesoscopic lattice model. Comput. Mater. Sci. 44(4): 1098-1106

Hafner S, Eckardt S, Luther T, Konke C 2006 Mesoscale modeling of concrete: Geometry and numerics. Comput. Struct. 84: 450-461

Hillerborg A, Modeer M, Petersson P E 1976 Analysis of crack formation and crack growth in concrete by means of fracture mechanics and finite elements. Cement and Concrete Res. 6: 773-782

Kouznetsova V, Brekelmans W A M, Baaijen F P T 2001 An approach to micro-macro modeling of heterogeneous materials. Computational. Mech. 27: 37-48

Kwan A K H, Wang Z M, Chan H C 1999 Mesoscopic study of concrete II: nonlinear finite element analysis. Comput. Struct. 70: 545-556

Leite J P B, Slowik V, Mihashi H 2004 Computer simulation of fracture processes of concrete using mesolevel models of lattice structures. Cement and Concrete Res. 34: 1025-1033

Lemaitre J 1987 Continuum damage mechanics theory and applications. Wien-New York Springer.

Mehta P K, Monteiro P J M 1993 Concrete: Microstructure, properties and materials. Indian Concrete Institute

Sadouki H, Wittmann F H 1988 On the analysis of the failure process in composite materials by numerical simulation. Mater. Sci. Eng. A104: 9-20

Schlangen E, Van Mier J G M 1992 Experimental and numerical analysis of micro-mechanisms of fracture of cement-based composites. Cement and Concrete Composites 14: 105-118

Schorn H, Rhode U 1991 Numerical simulation of crack propagation from microcracking to Fracture. Cement and Concrete Composites 13: 87-94

Shioya S 1967 On a semi-infinite thin plate with a circular inclusion under uniform tension. JSME 42: 623-636

Spagnoli A 2009 A micromechanical lattice model to describe the fracture behaviour of engineered cementitious composites. Comput. Mater. Sci. 46(1): 7-14

Van der Sluis O, Schreurs P J G, Brekelmans W A M, Meijer H E H 2000 Overall behaviour of heterogeneous elastoviscoplastic materials: Effect of microstructural modelling. Mechanics of Materials 32: 449-462

Van Mier J G M 1996 Fracture processes of concrete-assessment of material properties for fracture models. CRC Press

Vonk R A 1992 Softening of concrete loaded in compression. PhD thesis, Eindhoven University of Technology, The Netherlands

Wang Z M, Kwan A K H, Chan H C 1999 Mesoscopic study of concrete: generation of random aggregate structure and finite element mesh. Comput. Struct. Pergamon Press Ltd., 70: 533-544

Wriggers P and Moftah S O 2006 Mesoscale models for concrete: Homogenisation and damage behaviour. Finite elements in analysis and design 42: 623-636 